\section{Special-needs patients in pediatric dentistry: Progeroid syndrome. A case of dental management and oral rehabilitation}

\author{
Maria Grazia Cagetti, Nicole Camoni, \\ Flavia Cetraro, Massimo Scanferla, \\ Giacomo Maria Moretti
}

Department of Biomedical, Surgical and Dental Sciences, School of Dentistry, University of Milan, Italy

\begin{abstract}
This report presents a case of an eightyear-old girl affected by a progeroid syndrome of unclear genetic origins. The patient's dental history included oligodontia, premature deciduous exfoliation and roots abnormalities. She was treated with comprehensive oral rehabilitation using dentures. Oral health instructions were given during the whole treatment and follow-up period. The goal of improving the masticatory function and the esthetic was achieved, allowing the patient to increase her social abilities and self-confidence.
\end{abstract}

\section{Introduction}

Progeroid syndromes are a wide range of rare systemic diseases, which share significant anomalies in the orofacial region. ${ }^{1}$ Progeria is characterized by a phenotype that mimics a combination of premature aging and lower facial height. Patients suffering from this rare condition need comprehensive dental management because of their dental and skeletal anomalies.

The report presents a case of an eightyear-old girl affected by a progeroid syndrome of unclear genetic origin. The patient's dental history included oligodontia, premature deciduous exfoliation and roots anomalies.

\section{Case Report}

The child was born at 37 weeks with a Caesarean section. There was no consanguinity, history of miscarriages, significant maternal illnesses, or exposure to known teratogen. During the pre-birth ultrasound, reduced intrauterine growth for gestation age and globose abdomen were found. At birth she weighed $2250 \mathrm{~g}$ and she was 42 $\mathrm{cm}$ in height. A magnetic resonance image of the brain at 2 months from birth revealed brachycephaly, dural malformations and vascular developmental variations. She started talking at 10 months and she manifested a moderate psychomotor retardation. The patient was also diagnosed with hemangioma of the cephalic segment and of the back, scoliosis, skin xerosis, onychodystrophy with hypoplasia of the distal phalanx of the third finger and hypertrichosis. She also presents skin laxity and wrinkles of the neck and prominent eyebrows (Figure 1). Weight and height were below the $5^{\text {th }}$ percentile. These presenting features suggested a diagnosis of Petty syndrome. She was submitted to a comparative genomic hybridization array. No alterations were found in the patient array and in her parents. Nevertheless, as shown in Table 1, the child shared several characteristics with the Petty Syndrome (PLWPS), but she also presented some features similar to the HutchinsonGilford Progeria Syndrome (HGPS).

When the child presented for the first oral examination at the Dental Clinic of the University of Milan, she was 8 years old, 13 $\mathrm{kg}$ in weight and $110 \mathrm{~cm}$ in height. From a dental point of view, the mother referred a normal eruption timing of primary teeth, which quickly became darker and prematurely exfoliated. Consequently, the child was used to eat only soft foods, due to her difficulty in chewing hard foods, and needed a long time to eat her meals.

During the first clinical examination, an oligoblastodontia was suspected; orthopantomography displayed numerous agenesis of the permanent dentition, having less than half the normal number (oligodontia), delayed eruption, and misshapen teeth with radicular anomalies, compatible with a rizomicria as the roots were short and thin (Figure 1). No carious lesions were found; a wide degree of mobility of the two lower primary canines was discovered. Both thickness and height of the alveolar bone were extremely reduced in the upper and lower jaws. The condyles appeared dysmorphic and flattened. The child presented a swallow with lingual interposition and a class III occlusion in the rest position (Figure 1).

She was suggested to use a fluoridated tooth-paste (pea-size dose) and to carry out a modified Bass tooth-brushing technique at list 2 times per day after the main meals. The parents were invited to help the child during the home brushing. Professional hygiene and brushing instructions were provided during the first examination. The patient was calm and very cooperative. An informed consent was given to the parents
Correspondence: Maria Grazia Cagetti, Department of Biomedical, Surgical and Dental Sciences, School of Dentistry, University of Milan, Via Commenda 10, 20122 Milan (MI), Italy.

Tel.: +39.0250319008 - Fax: +39.0250319040 .

E-mail: maria.cagetti@unimi.it

Key words: Pediatric dentistry, progeroid syndrome, oral rehabilitation, dental anomalies.

Contributions: MGG did the literature research, has been involved in drafting the manuscript and reviewed the final manuscript. $\mathrm{NC}$, performed all preventive measures and has been involved in drafting the manuscript. FC, has taken the pictures and the radiographs. MS, designed the prosthesis and checked the dentures. GMM took the dental impressions.

Conflict of interest: the authors declare no potential conflict of interest.

Funding: none.

Received for publication: 25 November 2018

Revision received: 10 April 2019.

Accepted for publication: 10 April 2019.

This work is licensed under a Creative Commons Attribution NonCommercial 4.0 License (CC BY-NC 4.0).

(C) Copyright M.G. Cagetti et al., 2019

Licensee PAGEPress, Italy

Pediatric Reports 2019; 11:7951

doi:10.4081/pr.2019.7951

before the oral rehabilitation.

The solution proposed was a total removable prosthesis for the upper arch and a partial removable prosthesis for the lower arch (Figure 2).

The two prostheses were arranged in six appointments: 1) Preliminary alginate impressions: the smallest trays in commerce were used. 2) Fabrication of custom impression trays and secondary impressions: for the final impression a polyvinyl material was used. The element 5.3 was in eruption, for this reason a small groove in the tray was done to avoid over-compression on the site. 3) Boxing and pouring of secondary impression and fabrication of record bases: occlusion rims were used to obtain the correct vertical dimension. 4) Anterior artificial tooth arrangement: the upper elements were arranged from canine to canine, using the functional phoneme (especially words with /f/ and /s/) and protrusion and lateral movements in order to achieve the ideal position of the missing teeth. The facial references for symmetry and canine position were the wings of the nose. The child has been compliant during all the procedures. Laboratory 
made the teeth itself as the patient needs of teeth smaller than the commercial ones. 5) Posterior artificial tooth arrangement: even in this case the teeth were custom made in the laboratory, the correct position was arranged using bite and occlusion tests. 6) Final delivery.

The two dentures were manufactured simultaneously. Except for the groove for the element 5.3, the upper prosthesis does not have any significant difference with a standard total removable prosthesis.

The lower prosthesis was made using a plastic aligner on her natural teeth (elements 3.1, 3.2, 4.1, 4.2), with both mucosal and dental support. This strategy was chosen as a consequence of the reduced crest dimension and since normal anchorage using hooks was not possible due to the reduced stability of the permanent incisors affected by rizomicria. The aligner allows an axial load during chewing movements; this is the mechanical reason why a prosthesis with hooks was not suitable, as it would have produced a tangential load during chewing and a higher probability of traumatic avulsion of the child's natural teeth.

\section{Discussion}

The family of progeroid syndromes presents two main etiological factors: nuclear envelope alteration and gene mutation of DNA repair proteins. The hallmarks of these syndromes can be resumed in: metabolic defects, senescence, cell cycle deregulation, nuclear lamina alterations, epigenetic changes, telomere dysfunction, increased DNA damage, steam cell exhaustion and inflammation. ${ }^{2}$ Other progeroid syndromes with an unidentified genetic etiology as the Petty-Laxova-Wiedemann Progeroid Syndrome (PLWPS) are reported. ${ }^{3}$

Nevertheless, each syndrome presents its own peculiar clinical features. ${ }^{2}$ The Hutchinson-Gilford Progeria Syndrome (HGPS) is the most common and studied type of progeria. It is characterized by alopecia, atherosclerosis, prominent scalp veins, lipodystrophy, micrognathia and diminished joint range of motion. Subjects with PLWS have, as for other syndromes, severe pre- and post-natal growth retardation, progeroid face, large open fontanelle in infancy, umbilical hernia at birth, pseudomacrocephaly, wide calvaria, sparse scalp hair, markedly diminished subcutaneous fat, but they also present scoliosis, partial cutaneous syndactyly, aplastic and hypoplastic distal phalanges with aplasia and hypoplasia of nails and normal cogni- tive and motor development. $3-5$

Literature offers mainly case reports of PLWS and HGPS. The estimated prevalence of newborns affected by progeroid syndrome is 1 in 4 million; this hypothetical number includes the misdiagnosis of the wide range of progeroid syndromes.

In all these syndromes the oral apparatus is highly affected. The upper and lower maxilla present variations that are difficult to handle and the frequent dental anomalies impose an accurate study of each patient. 5,6 Developmental defects of the enamel, anomalies in teeth number, position or shape, malocclusion, natal teeth or delayed eruption are reported. For these patients the first dental examination should be planned as soon as the first deciduous tooth erupts in order to promote a tailored preventive plan. The oral rehabilitation can be realized even in pre-school children, timing and techniques change depending on the patient collaboration and number of missing teeth. Cases of oral prosthetic rehabilitation in little children affected by genetic conditions, or after teeth extraction for trauma or early childhood caries are reported in literature. 7,8 Standardized protocols are not available; each solution is tailored according to the patient condition and the state of art of the prosthetic technology.

The initial dental management of this case report was based on maintaining oral health, giving oral hygiene instructions to

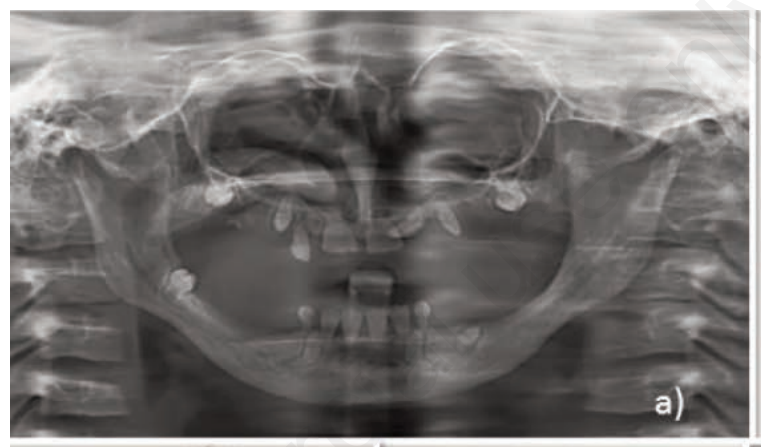

a) Orthopantomography b $1,2,3)$ Frontal picture, profile and teleradiography without prosthesis c $1,2,3)$ Frontal picture, profile and teleradiography with prosthesis
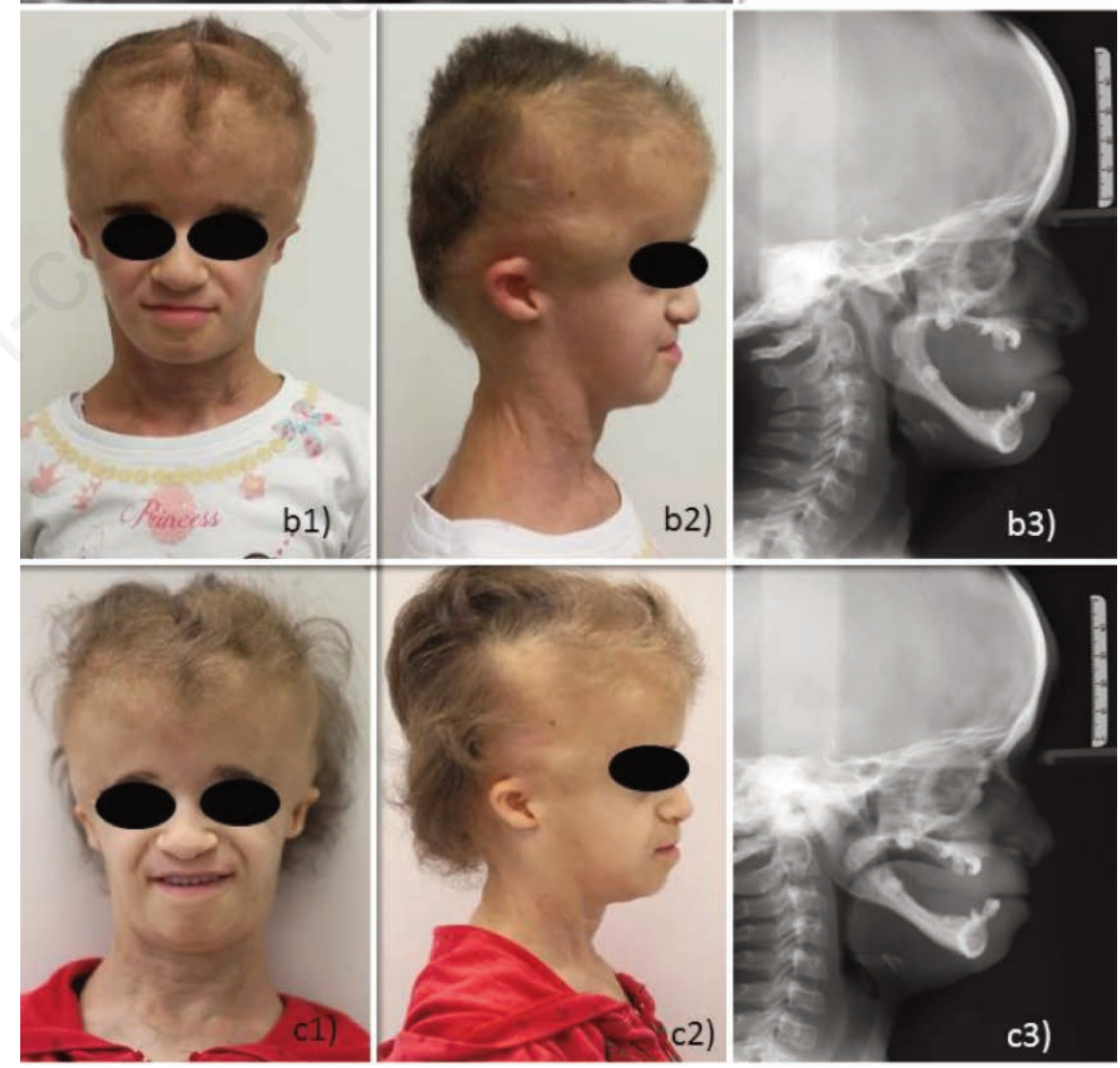

Figure 1. X-rays and pictures before and after the oral rehabilitation. 
the girl and her parents. The prosthetic rehabilitation was challenging as the child presented reduced mouth opening, ipomaxillia and fibrous and floating gum tissue. She showed a consistent tendency to protrusive posture that is a disadvantageous feature for the prosthesis realization and use.

At the clinical check the girl presented the elements 7.3 and 8.3 with a third degree of mobility according to the Miller mobility index (more than $1 \mathrm{~mm}$ of horizontal movement and depressible within the socket). ${ }^{7}$ For this reason, both teeth were extracted, while 5.3 was left in situ since it did not cause impediments to the rehabilitation. The presence of teeth in osseous and mucosal inclusion suggested the necessity of consistent maintenance including denture renewals. The teeth of the dentures were $a d$ $h o c$ manufactured in the dental laboratory

Table 1. Patient features compared to the Hutchinson-Gilford Progeria Syndrome (HGPS) and the Petty Syndrome (PWLS).

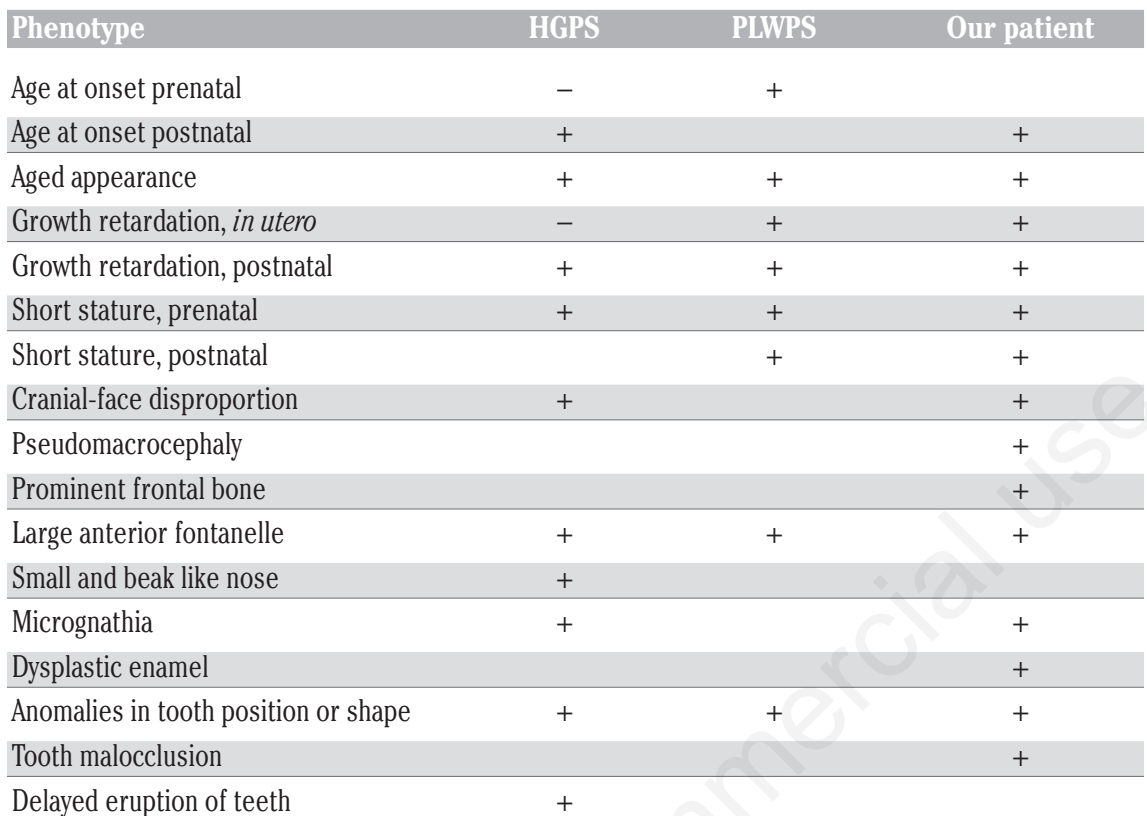

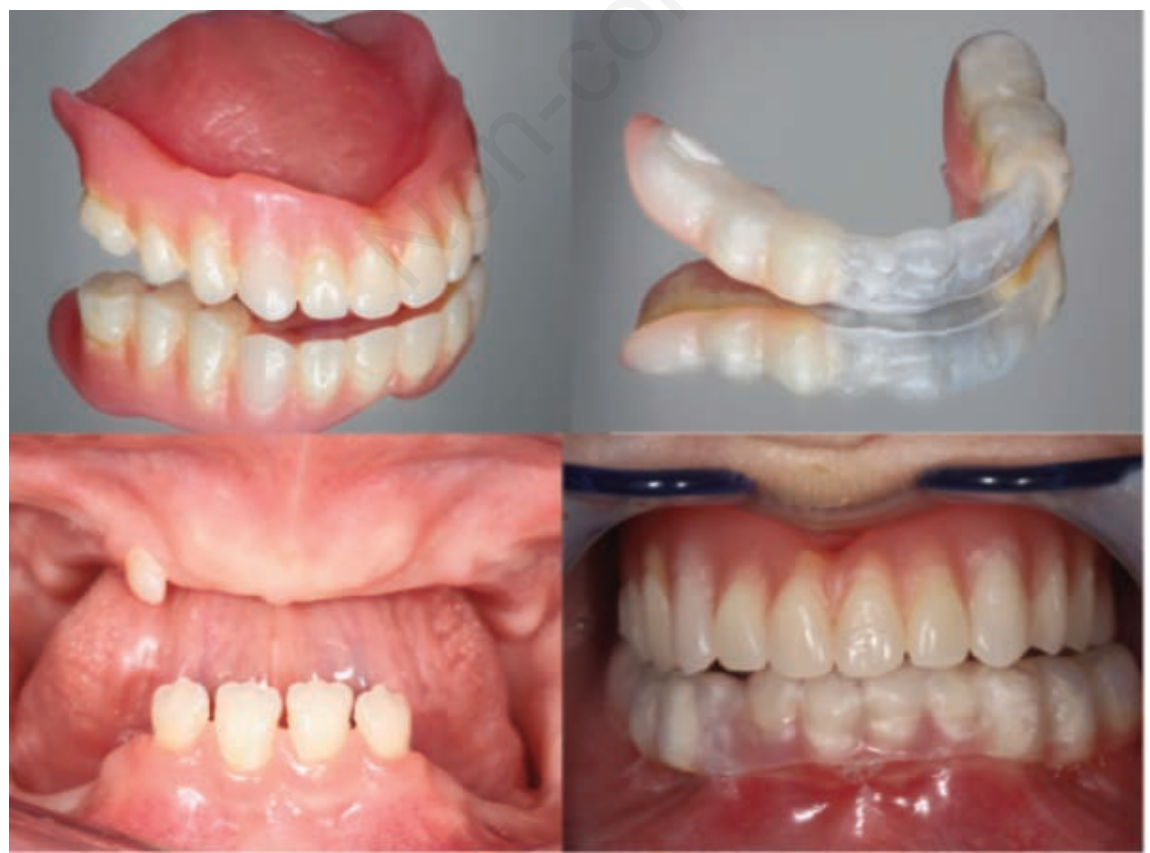

Figure 2. Upper and lower dentures and intra-oral pictures before and after the insertion of the dentures.

since the patient needed dental elements smaller than those available on the market (Figure 2). Before delivering, a comprehensive test of usage was carried out in order to check compression sites of the oral mucosa. Figure 1 shows the pictures of the face in frontal and lateral views before (b1 and b2) and after ( $\mathrm{c} 1$ and $\mathrm{c} 2)$ prosthetic rehabilitation, the panoramic radiograph and the two latero-lateral teleradiographs, without wearing (b3) and wearing the dentures (c3). In Figure b3, due to the absence of teeth, the child was not able to occlude and consequently used her tongue to maintain the anterior vertical dimension; in Figure c3, after wearing the dentures, the child was able to reach a physiological rest position of the mandible.

During the appointments following the prosthesis delivery, all interferences of the prosthesis were trimmed with a low speed handpiece. Natural superior teeth (1.1 and 2.1) showed an eruptive sprint as they were stimulated by the prosthesis. From the first day following the delivery, the girl could eat faster and bite hard foods properly. Being capable of eating the same food of her family and peers benefitted her social life and self-confidence; she became more talkative and her parents reported an overall improvement of the quality of her life. The child was so happy with her dentures, that she also wore them at night-time, despite she was suggested to use them only during the day. The patient is nowadays re-evaluated every two months in order to prevent oral mucosa alterations, to control natural teeth stability and to adjust the prostheses in order to endure teeth eruption. The longterm implications of this case include denture renewal when maxillary bones will grow, especially during the pubertal spurt. The future goal will be the preservation of her natural teeth, giving her comfort and stability during daily activities. The prostheses will be updated according to the new techniques that will be developed, moreover, comfortable fixed solution using implants will be probably proposed in the future. 8

\section{Conclusions}

This is certainly a challenging case. Few dentists with different specialties cooperated to achieve an optimal prosthetic comfort for the young girl. The goal of improving the masticatory function and the aesthetic was obtained, allowing the patient to increase her social abilities and self-confidence. The actual and future aim is to follow her growth, modifying the dentures 
when necessary, and preserve her natural teeth during the next years.

\section{References}

1. Sinha JK, Ghosh S, Raghunath M. Progeria: a rare genetic premature ageing disorder. Indian $\mathrm{J}$ Med Res 2014;139:667-74.

2. Carrero D, Soria-Valles C, López-Otín C. Hallmarks of progeroid syndromes: lessons from mice and reprogrammed cells. Dis Model Mech. 2016;9:719-35.
3. Wiedemann HR. An unidentified neonatal progeroid syndrome: followup report. Eur J Pediatr 1979;130:6570 .

4. Rastogi R, Chander Mohan SM. Progeria syndrome: a case report. Indian J Orthop 2008;42:97-9.

5. Delgado-Luengo WN, Petty EM, SolísAñez E, et al. Petty-LaxovaWiedemann progeroid syndrome: further phenotypical delineation and confirmation of a rare syndrome of premature aging. Am J Med Genet A 2009;149A:2200-5.
6. Ullrich NJ, Silvera VM, Campbell SE, Gordon LB. Craniofacial abnormalities in Hutchinson-Gilford progeria syndrome. AJNR Am J Neuroradiol 2012;33:1512-8.

7. Dimova-Gabrovska M, Dimitrova D, Mitronin VA. Removable prosthetic treatment in children - literature review. Journal of IMAB 2018;24:2172-6.

8. Schnabl D, Grunert I, Schmuth M, Kapferer-Seebacher I. Prosthetic rehabilitation of patients with hypohidrotic ectodermal dysplasia: A systematic review. J Oral Rehabil 2018;45:555-70. 